\title{
Coupled Active Thermography and Terahertz Technique in Composite Materials Defects' Evaluation
}

\author{
by B. Szymanik, P. Lopato, G. Psuj
}

Department of Electrical and Computer Engineering, Faculty of Electrical Engineering, West Pomeranian University of Technology, al. Piastów 17, 70-310 Szczecin, Poland, szymanik@zut.edu.pl

\begin{abstract}
In this paper the technique of defects evaluation in composite materials based on a combination of active thermography method and terahertz technique is presented. The composite glass fiber sample with delaminations produced by load conditions was chosen for the tests. Active Infrared thermography with halogen lamps and terahertz technique are sensitive to changes in different physical parameters of the tested material. The combination of these techniques, performed by the data fusion of obtained measurements results allows to obtain the full information on the detected material's defect.
\end{abstract}

\section{Introduction}

Modern, lightweight, easy-to-use and extremely durable composite materials accelerated the development of some branches of industry. The new materials are used in, for example, aircraft or ships hulls construction and as the core of the wind turbine blades. These constructions are subjected to heavy loads during usage, which can result in a significant weakening of the structure, therefore, require a continuous monitoring, using non destructive testing (NDT) techniques for early detection of possible defects. The ultrasonic testing, radiography and shearography are common methods of composite materials non-destructive evaluation. In this paper the composite sample with artificial flaws (drill holes) was chosen for the tests.

Two different methods of NDT has been used to examine the presented sample. The active infrared thermography with halogen lamp heating allows the fast and global evaluation of tested material and is widely used as reliable NDT method for many materials [1]. The method is however quite limited when it comes to detect the relatively small defects in materials with low values of thermal conductivity. The second used method - terahertz technique, from the other hand, is sensitive for changes of refractive index, therefore it is very well suited for composite materials evaluation. This method, however should be considered as local one, since it requires point by point material inspection.

First, the experimental methods and examined sample are presented. The material's evaluation was performed separately using each method. The results of image processing for IRT and signal parameterization are presented. Finally, to obtain the possibly full information about the detected defects, IRT and Terahertz technique were combined, by using the data fusion method.

\section{Experimental methods}

It has been shown, that the composite materials can be examined by using active thermovision (IRT - InfraRed Technique) [2 - 4]. In this method of non destructive testing the initial thermal non equilibrium of the system has to be induced. The temperature difference in examined samples can be produced using several methods of excitation. In this article the active thermography with halogen lamps as external energy source is used. The sample is heated from the back side (transition mode) or the front side (reflection mode) and observed simultaneously by the thermovision camera. The free flow of heat in the examined material is highly affected by its internal defects, and thus it is possible to detect them by the temperature distribution at the sample's surface. Detected flaws are visible as the overheated or under heated spots and their contour corresponds to the real defect shape. Therefore active thermovision can be used to detect all kinds of defects widely seen in modern composite materials, like delaminations, inclusions, impact damage, holes, etc. The main advantage of this method is its speed and global character. The large areas can be evaluated at once, and the results are obtained almost online.

The second method of NDT used in this study is terahertz technique. The electromagnetic radiation in terahertz frequency range (0.1-10 THz) enables non-invasive, non-ionizing and non-contact examination of dielectric composite structures. The terahertz NDT is sensitive for changes of refractive index $n$, thus any defect which noticeably disturbs refractive index can be detected (e.g. void, delamination, inclusion, fiber/matrix distribution, surface roughness, fiber waviness, internal interfaces between layers). In most cases, defects are located by reflection or transmission imaging based on pulsed terahertz TDS (Time Domain Spectroscopy) [5 - 8]. In this technique pico-second electromagnetic pulses are generated, focused by $\mathrm{THz}$ optics on examined object and received by measuring system. The method is well 


\subsection{1/qirt.2016.040}

suited for evaluation of layered materials. Each interface between separate layers causes reflection of incident THz pulse and attenuation of transmitted one. Differences in delays of the propagated pulses and their echo (delayed layer reflections) enable characterization of the thicknesses and inner structure state. Very short pulses contain wide frequency bandwidth $(0.05-3 \mathrm{THz})$ and therefore it is possible to carry one single point broadband measurements.

\subsection{Composite sample}

The sample made from reinforced glass-fiber composite with $85 \mathrm{~mm} \times 50 \mathrm{~mm} \times 5 \mathrm{~mm}$ dimensions was used in this study. To test the capabilities of each used NDT method the set of artificial defects in the form of drilled holes of different diameters, depth and aliment within the sample was prepared. The photo and the approximate geometry (the real positions and sizes of defects are slightly different from those indicated in the technical drawing) of prepared sample is shown in the Fig. 1. Two general sets of defects are present: 12 holes of different depth (from $2 \mathrm{~mm}$ to $4 \mathrm{~mm}$ ) and diameters (from $1 \mathrm{~mm}$ to $4 \mathrm{~mm}$ ) and 21 back side bores with $1 \mathrm{~mm}$ diameter located at different depths. The bores are located close to each other, the minimum distance between two bores is $1 \mathrm{~mm}$, to check the methods resolution. The sample was covered in black paint in order to prepare it to the IRT experiment. It was also prepared to the final fusion operation by using the conducting foil to mark some points important for further analysis.

a)

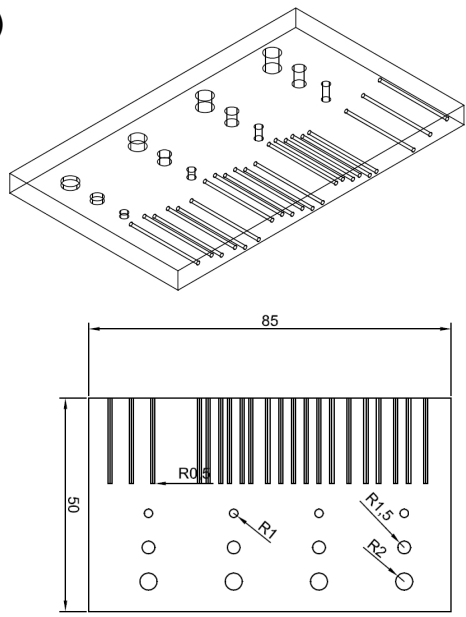

b)



c)

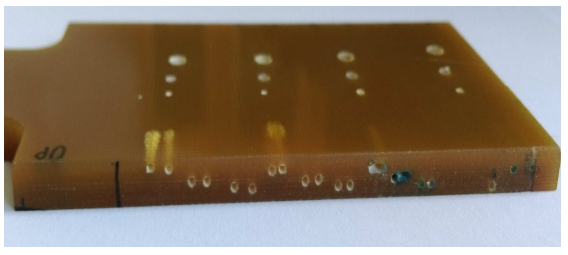

Fig. 1 Evaluated glass-fiber reinforced composite sample: a) geometry of the sample, b) photo of painted sample with markers, c) photo of raw sample with artificial defects.

\subsection{Experimental setups}

Active thermography inspection was performed using halogen lamp excitation (max $P=2000 \mathrm{~W}$ ) and thermovision camera FLIR A325. As it is shown in Fig. 2a, the sample was heated from the back side, and observed from the front (transmission mode). In this setup the deeper defects should be visible more accurately, since the heat will accumulate in reduced material volume between the surface and the defect end. Also, the sample is highly damaged in the region of back side bores (because of high concentration of defects some delamination also occurred), an it also should be possible to detect with this setup. Since the thermal diffusion coefficient has the low value (comparing to conducting materials) the time of heating had to be respectively long. The step pulse method was used, the length of the pulse was set to $60 \mathrm{~s}$. After this time the sample was observed for 300 more seconds. The recording frequency was set to $1 \mathrm{~Hz}$ (1 image per second). As a result two sequences: heating phase (with 60 thermograms) and cooling phase (with 300 thermograms) were obtained.

Terahertz inspection was performed using imaging system based on TRay4000 pulsed spectroscope of Picometrix. Measuring head was scanned over the surface of a sample using 2D positioning system, and for each $(x, y)$ position time domain signal $s(t)$ was acquired. Time information is correlated with depth in examined material, thus using this method depth dependent information about defects can be obtained (eg. between which layers there is a delamination). Pico-second electromagnetic pulses are generated by photoconductive antenna (PCA), focused by $\mathrm{THz}$ optics (3" HDPE focusing lens) on examined object and after interaction - acquired by PCA based receiver head. In order to obtain the case of normal incidence, beam splitter was utilized as shown in Fig. $2 \mathrm{~b}$. 
a)

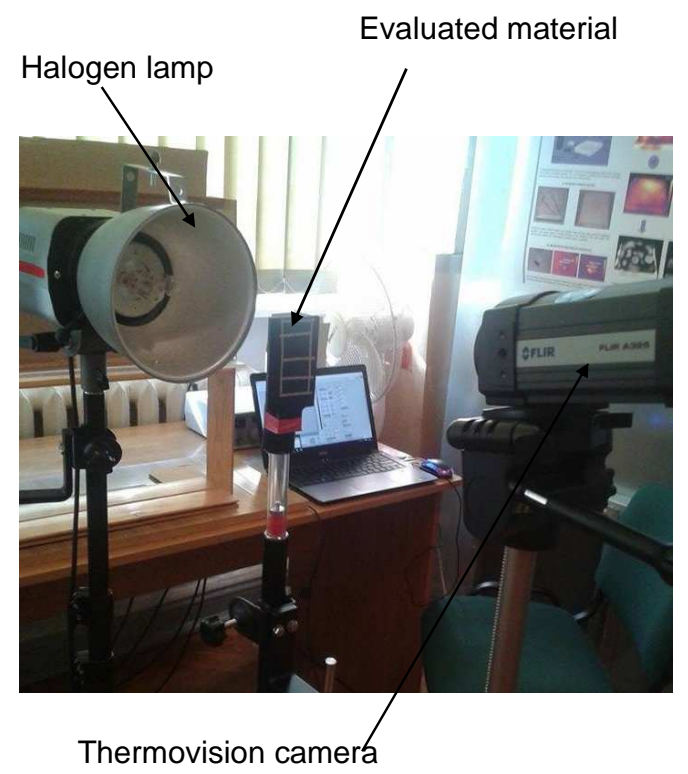

b)

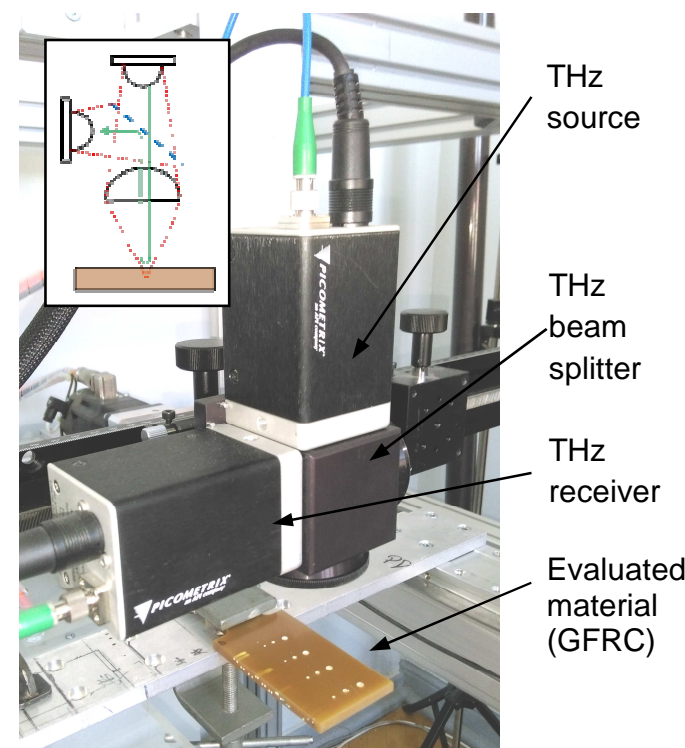

Fig. 2 Photos of measuring setups: a) IRT system, b) terahertz inspection system

\section{The experiments' results}

\subsection{IR testing results}

The small sizes of defects make it very difficult to receive the proper quality of the results using themovision method. To improve the defects' visibility several methods of image processing were used. First the temperature inhomogeneity produced by the not perfect uniform illumination of the sample by the halogen lamp had to be removed. In case the sample with small defects it is convenient to use the median 2D filter with large mask (larger than maximum defect's size) to approximate the non uniform heating influence itself, and then subtract this approximation from the original image to obtain better quality result. This simple operation is shown in Fig. $3 \mathrm{~b}$. Then, we applied the thermal contrast function, defined as follows [2]:

$$
O_{T}(i, j, t)=\frac{I_{T d}(i, j, t)-I_{T d}(i, j, t=0)}{I_{a s a}(t)-I_{a s a}(t=0)}
$$

where $O_{T}(i, j, t)$ - output temperature value in every point, $I_{T d}(i, j, t)$ - input temperature value in every point defined as possible defected, $I_{\text {asa }}(t)$ - Input temperature averaged over the entire area defined as not defected. $t=0$ indicates the first image in the sequence. This procedure enables to sharpen the output images, as shown in Fig. 3c.

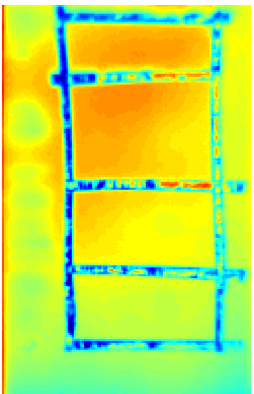

a)

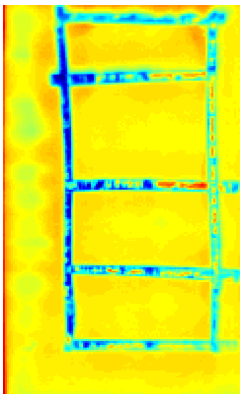

b)



c)

Fig. 3 a) exemplary raw thermogram of the evaluated sample, b) thermogram after the median filtering procedure, $c$ ) image after contrast computation 


\subsection{1/qirt.2016.040}

Additionally the first derivative of the processed data over the time domain from the heating phase and the second derivative from the cooling phase were computed. Derivative represents of the rate of function change, therefore it can be used to enhance the visibility of the defects, which can be noticed in Fig. 4.

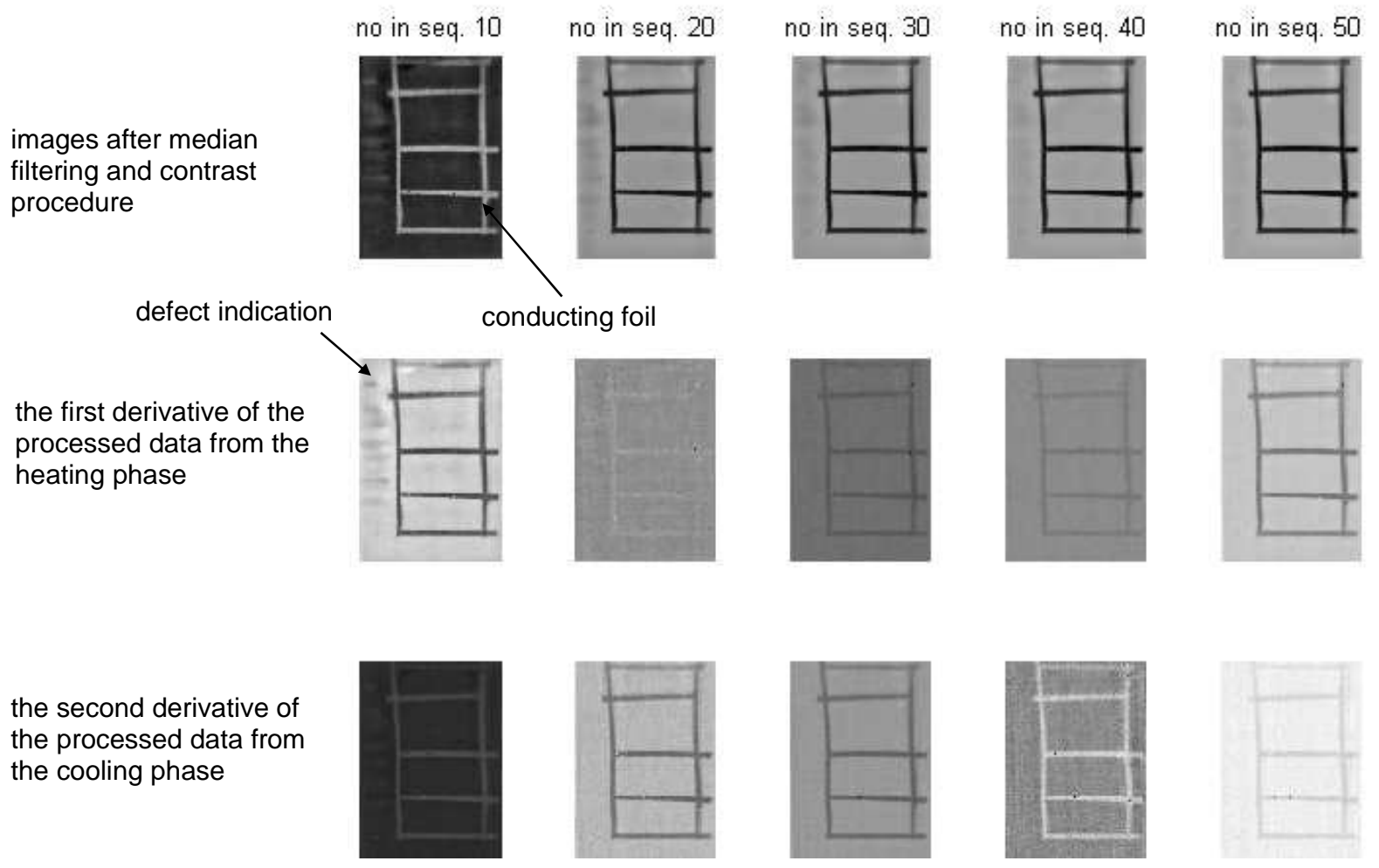

Fig. 4 Chosen results of image processing of obtained thermograms

As it can be seen in the Fig only few images from the sequence can be used to evaluate the sample. The best results along with the data obtained using DFT (Discreet Fourier Transform) were carefully chosen and used in the data fusion procedure.

\subsection{THz testing results}

The exemplary results of $\mathrm{THz}$ measurements are shown in Fig 5. For each $(x, y)$ position time domain signal $s(t)$ was acquired. In order to simplify further processing, the parametrization of $s(t)$ will be carried out. One can observe, the changes of the signal caused by defects existence are depth dependent and hard to evaluate because of complicated background (the time response consists of many pulses reflected off many layers existing in examined composite material), thus a set of features for further processing should be carefully defined.

a)

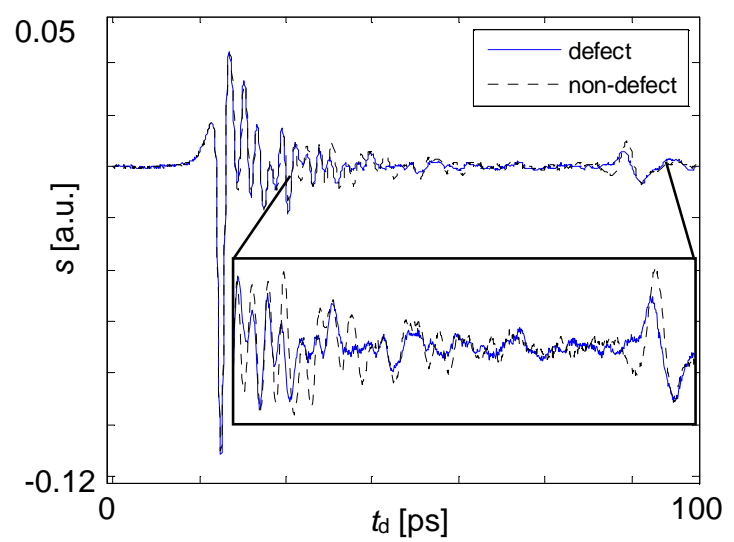

b)

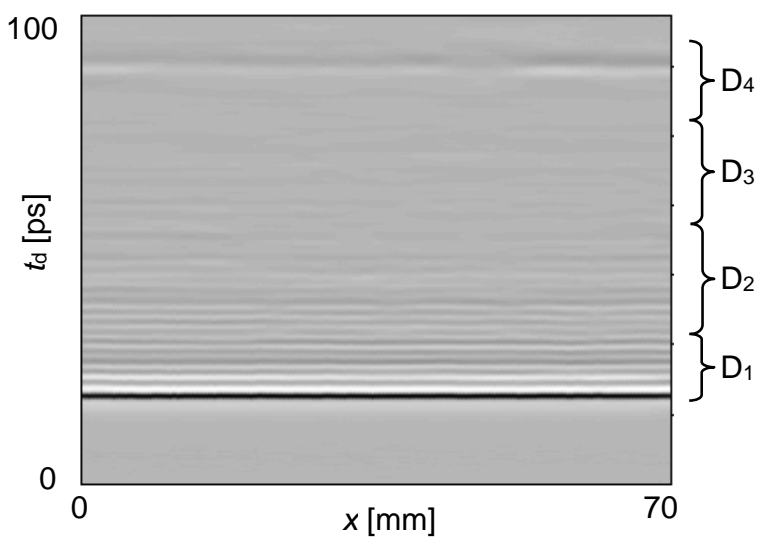

Fig. 5 Exemplary signals obtained using THz inspection: a) A-scan, b) B-scan. 


\subsection{1/qirt.2016.040}

In order to calculate a features set the time response (A-scan) was divided on four parts (time gates) as shown in Fig. 5. $D_{1}$ and $D_{4}$ correspond to front and back surface of the evaluated material respectively, while $D_{2}$ and $D_{3}$ correspond to interior part of the sample. For each of time gates the following set of features were defined:

$$
\begin{gathered}
\xi_{D n, 1}=\max \left[s_{D n}(t)\right] \\
\xi_{D n, 2}=\min \left[s_{D n}(t)\right] \\
\xi_{D n, 3}=\max \left[s_{D n}(t)\right]-\min \left[s_{D n}(t)\right]
\end{gathered}
$$

$$
\begin{aligned}
\xi_{D n, 4} & =\int_{t_{1}}^{t_{2}}\left|s_{D n}(t)\right| d t \\
\xi_{D n, 5} & =\operatorname{var}\left[s_{D n}(t)\right] \\
\xi_{D n, 6} & =\int_{f_{1}}^{f_{2}}\left|S_{D n}(f)\right| d f
\end{aligned}
$$

where: $\max []$ - maximum value, $\min []$ - minimum value, $\operatorname{var}[]$ - variance, $n=1 . .4-$ time gate number, $s_{D n}(t)-$ time domain signal in given $D_{\mathrm{n}}$ time gate, $S_{\mathrm{Dn}}(f)$ - frequency domain signal in given $D_{\mathrm{n}}$ time gate. Exemplary distributions of proposed features in case of interior defects are presented in Fig. 6.
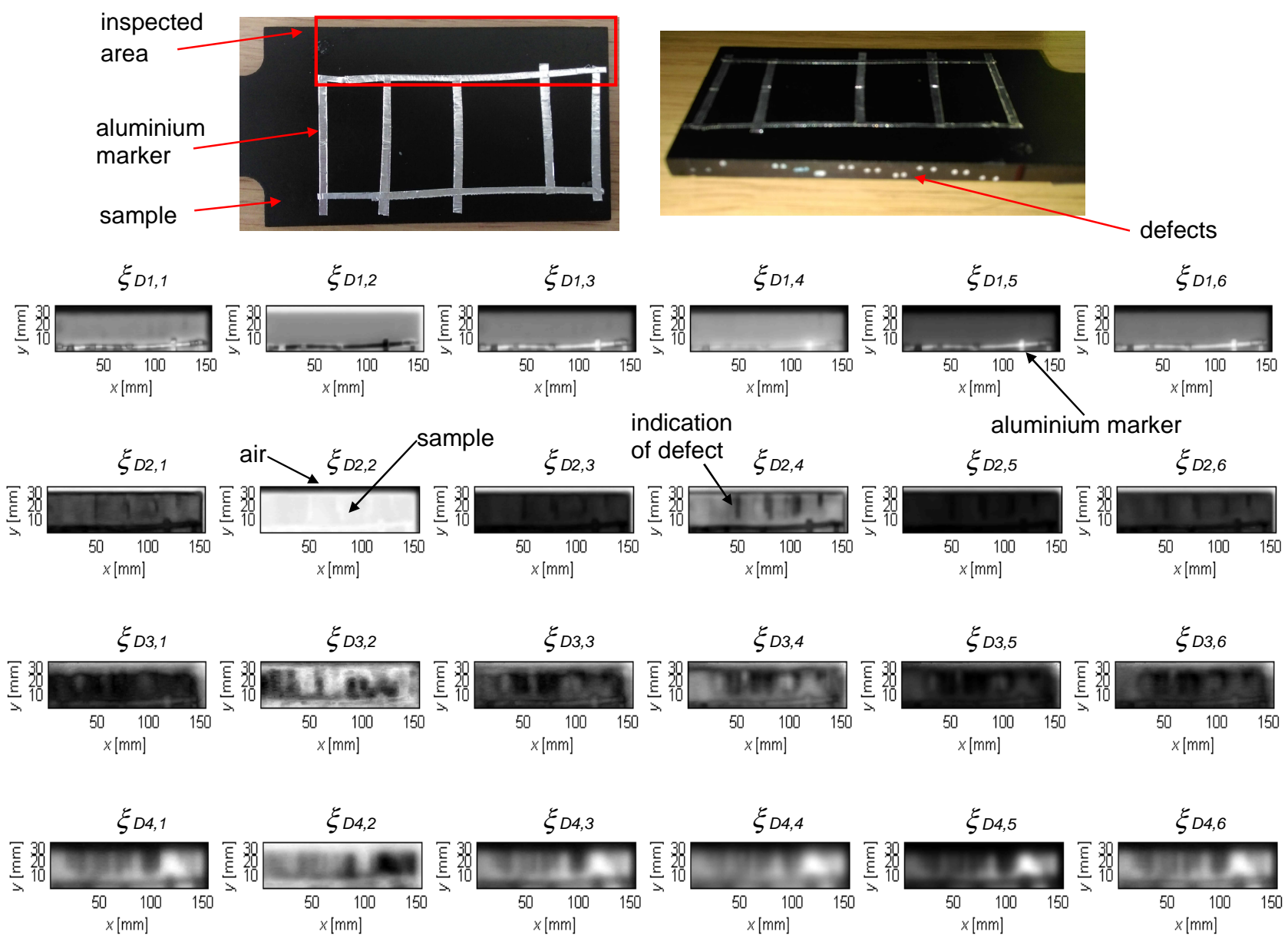

Fig. 6 Exemplary 2D distributions of proposed parameters in case of interior defects.

For time gate $\mathrm{D}_{4}$, resulting parameters distributions are blurred (with unknown point spread function, PSF) because of wave scattering in irregular structure of evaluated material and dispersion. In order to obtain higher spatial resolution the procedure based on blind deconvolution was utilized. The deconvolution procedure uses maximum likelihood algorithm. Thus, the additional feature was proposed based on $\xi_{D 4,5}$ feature:

$$
\xi_{D 4,7}=\text { blindDecon }\left[\operatorname{var}\left[s_{D 4}(x, y, t)\right]\right]
$$




\subsection{1/qirt.2016.040}

where: blindDecon[] - blind deconvolution procedure. Results of proposed procedure are shown in Fig.7. One can see, utilization of blind deconvolution procedure enabled more detailed observation of defects based on parameters obtained from back surface response.

Finally, we proposed 25 initial features from pulsed terahertz inspection. They will be utilized in data fusion procedure presented in next section.



Fig. 7 Results of blind deconvolution operation: raw data (left side) and processed data (right side).

\section{Data Fusion}

None of obtained parameters distributions can support one with complementary information about the structure state of the material. On the other hand, even within a single method different features of acquired data can provide one with unique information pertaining to current stage of the material's structure, i.e. time response allow to monitor material's condition at different depth. Therefore, in order to fully conduct the process of nondestructive imaging of the structure state data fusion of multiple features extracted from both methods results were carried out. The multiple sources inspection make the evaluation system more robust for the unwanted disturbances and increase the overall performance of the accession process [9], [10]. The block diagram of the algorithm utilized in this paper is presented in Fig. 8

First, all parameters distributions achieved from both methods were spatially registered. In order to carry out the process the metallic markers detectable by both methods were utilized during inspection procedures. The edges of the marker lines were used to extract and define the pairs of control point in both methods data spaces. The photo of the sample was used as a reference data. In order to transform the parameters distribution into common representation format a standard image geometrical perspective transformation model was used in the registration process. The spatial relationship between the successive pairs of control points in distributions achieved by both methods was used to calculate the coefficient of the transformation model. The exemplary results of the data registration process in presented in Fig. 9. After transformation, the distributions of parameters were cut into five parts representing different group of defects $\left(G D_{1}-G D_{5}\right)$.



Fig. 8 The stage diagram of the applied multiparameters data fusion procedure. 
a)

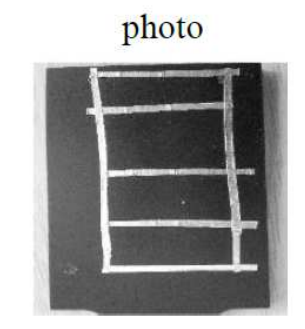

b)

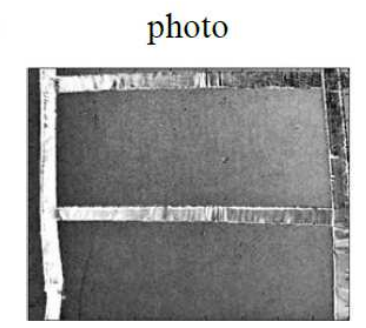

$\mathrm{THz}$ inspection result

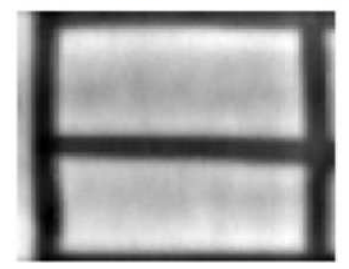

$\mathrm{THz}$ inspection result

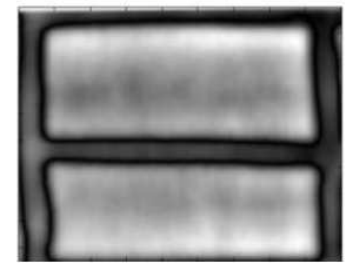

IRT inspection result



IRT inspection result



Fig. 9 Data registration results: a) imput signals parameters distributions and a photo of the sample, b) transformed photo and inspection results into common representation resulting data space.

After the data registration process the features distributions obtained for each single method were analyzed and preselected. During the preselection process two aspects were taken into account: first to minimize the number of similar information constancy and second to preserve the possibility of observation of different aspects of structure state assessment. Finally the set of 10 features representing both methods were used to define the features' vector for processing of the data fusion algorithm. The exemplary results obtained for defects GD1 are shown in Fig. 10. Next, in order to combine information content of different features and to reduce the dimensionality of each GD database, a vector data transformation procedures were applied. The principal component analysis (PCA) was carried out to reduce the number of features providing similar information about the material degradation stage [11]. As a result a space of
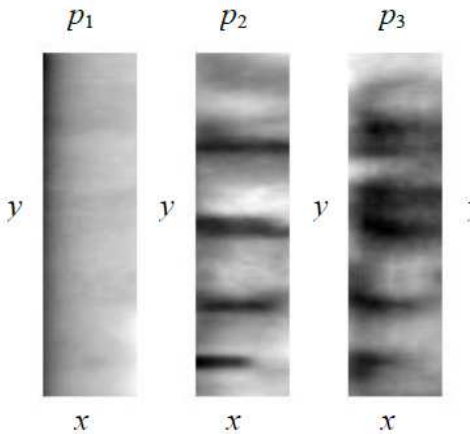

$x$



$x$

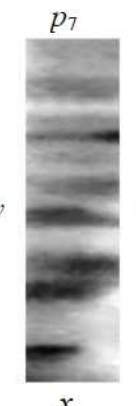

$x$

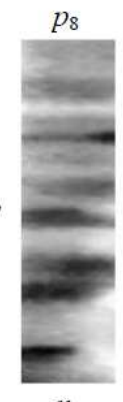

$x$

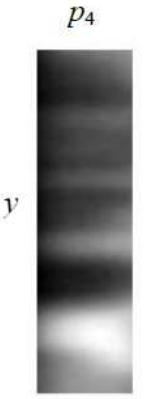

$x$

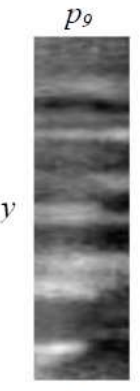

$x$

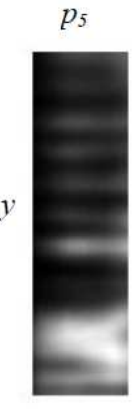

$x$

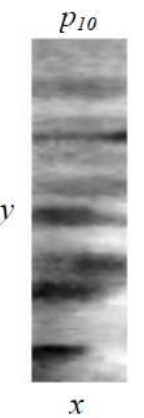

Fig. 10 Distributions of selected features representing inspection results THz ( $\left.p_{1}-p_{5}\right)$ and IR ( $\left.p_{6}-p_{10}\right)$ inspection results: $p_{1-}$ $p_{4}$ represents parameter $\xi_{\mathrm{Dn}, 6}$ for successive time gates $D_{1}-D_{4}$, $p_{5}$ represents parameter $\xi_{04,7}, p_{6}-p_{8}$ represents amplitude, thermal contrast and data after median filtering distributions in the second time step of the heating stage, $p 9$ and $p_{10}$ represents derivative of the temperature at the beginning of the cooling stage and heating stage respectively. 


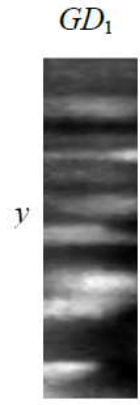

$x$
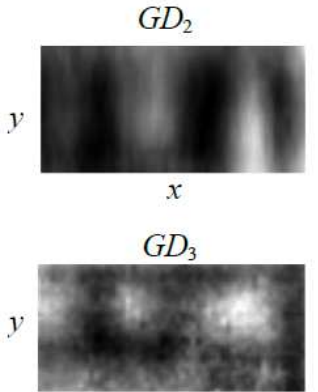

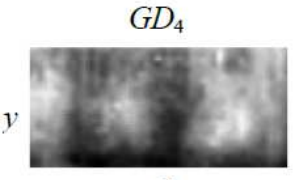

$x$



$x$

Fig. 11 Distributions of selected features representing inspection results $\mathrm{THz}\left(p_{1}-p_{5}\right)$ and IR ( $\left.p_{6}-p_{10}\right)$ inspection results: $p_{1}-$ $p_{4}$ represents parameter $\xi_{\mathrm{Dn}, 6}$ for successive time gates $D_{1}-D_{4}$, $p_{5}$ represents parameter $\xi_{\mathrm{D} 4,7}, p_{6}-p_{8}$ represents amplitude, thermal contrast and data after median filtering distributions in the second time step of the heating stage, $p 9$ and $p_{10}$ represents gradient of the temerature between at the beginning of the cooling stage and heating stage respectively.

A new variables $p c$ (principal components), which are a linear combination of the original features and are sorted in accordance to the variance level of each $p c$, were achieved. Finally, two components representing the highest variance were utilized for further analysis.

After the new components extraction multiresolution analysis (MRA) was carried out to obtain final data fusion results [10] In this paper the wavelet decomposition WT was utilized. WT is one of the most effective decompositions methods allowing to obtain a good resolution in both time and frequency domain. The results of the MRA data fusion were presented in Fig. 11. The idea of fusing the data into single image is to achieve more complementary information in simple representation format. The application of MRA data fusion allows to preserve information of both high and low spatial frequency characteristic in one common distribution. Therefore the nondestructive imaging of composite's structure condition can allow to achieve higher confidence in decision making process. The advantage of the integration procedure can be noticed in case of $G D_{1}$ defects area inspection. Those defects were drilled along and between the successive layers of fibers. As a result a holes were produced with degradations in surrounding neighborhood. The single inspection methods allows to visualize the drilled defects (Fig. 10), however the impact of the delamination on the distributions of the parameters is not clearly observed. The described effects of holes surroundings can be noticed in the fused results (Fig. 11). The Holes are depicted by white bands with high intensity, while the delaminations are represented by lower intensity of color.

\section{Conclusions}

Composite materials' evaluation is a difficult task, and there is no doubt that the methods which can be used in these structures' testing still need to be developed. Complex, layered inner structure of modern composites results in complicated response, even with no defect cases. Therefore, the multi sensor approach seems to be the proper choice. Using methods which are complementary to each other and sensitive for different materials' properties changes may significantly improve the evaluation results. The results obtained using different methods can be successfully combined using data fusion algorithms.

In this article the two methods of NDT were used to evaluate the composite sample with a number of small defects. In case of IR technique such defects are very difficult to detect in the materials with the low values of thermal conductivity. The results obtained using active thermovision can be compared with those obtained with terahertz technique. The latter is sensitive to changes of refractive index, therefore it is extremely useful in evaluation of layered materials. Finally the results of material inspection using both methods, after the registration process, were combined. The results of data fusion are interesting especially in case of the side bores, where combining the methods gave information about the defects location and also shown the material degradation in a form of delamination.

\section{REFERENCES}

[1] Kapadia, Non Destructive Testing of Composite Materials, TWI Ltd

[2] Maladegue X., Theory and practice of infrared technology for nondestructive testing. JohnWiley and Sons, 2001

[3] D. Balageas· X. Maldague - D. Burleigh · V. P. Vavilov ·B. Oswald-Tranta - J.-M. Roche · C. Pradere · G. M. Carlomagno, Thermal (IR) and Other NDT Techniques for Improved Material Inspection, J Nondestruct Eval (2016) $35: 18$ 


\subsection{1/qirt.2016.040}

[4 ] R. Sikora, T. Chady, B. Szymanik, Infrared Thermographic Testing of Composite Materials with Adhesive Joints, in proceedings of 18th WCNDT, 16-20 April 2012, Durban, South Africa

[5 ] Mittelman D. M., Gupta M., Neelamani R., Baraniuk R. G., Rudd J. V., Koch M., Recent advances in terahertz imaging, Applied Physics, Lasers and Optics, B 68 (1999), 1085 - 1094.

[6 ] Lopato P., Chady T., Terahertz detection and identification of defects in layered polymer composites and composite coatings, Nondestructive Testing and Evaluation, vol. 28, iss. 1, 2013.

[7 ] Palka N., Miedzińska D.: Detailed non-destructive evaluation of UHMWPE composites in the terahertz range, Optical and Quantum Electronics, 46, 2014, pp. 515-525.

[8 ] Mittelman D., Sensing with terahertz radiation, Springer, Berlin, 2010.

[9] C. Kohl, M. Krause, C. Maierhofer and J. Wöstmann, 2D- and 3D-visualisation of NDT-data using data fusion technique, Materials and Structures, 38, 2005, pp. 817-826

[10 ]Z. Liu et al., Survey: State of the Art in NDE Data Fusion Techniques, IEEE Transaction on Instrumentations and Measurements, vol. 56, no.6, 2007, pp. 2435-2436

[11 ]C. Wang et al., Principal component analysis based three-dimensional operational modal anlysis, International Journal of Applied Electromagnetics and Mechanics 45 (2014), pp. 137-144 